\title{
Predicting pathological axillary lymph node status with ultrasound following neoadjuvant therapy for breast cancer
}

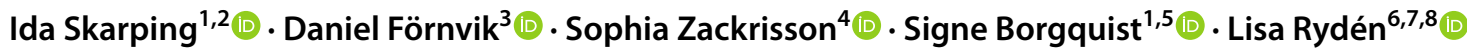

Received: 27 January 2021 / Accepted: 1 June 2021 / Published online: 12 June 2021

(c) The Author(s) 2021

\begin{abstract}
Purpose High-performing imaging and predictive markers are warranted to minimize surgical overtreatment of the axilla in breast cancer $(\mathrm{BC})$ patients receiving neoadjuvant chemotherapy (NACT). Here we have investigated whether axillary ultrasound (AUS) could identify axillary lymph node (ALN) metastasis (ALNM) pre-NACT and post-NACT for BC. The association of tumor, AUS features and mammographic density (MD) with axillary-pathological complete response (axillarypCR) post-NACT was also assessed.

Methods The NeoDense-study cohort ( $N=202$, NACT during 2014-2019), constituted a pre-NACT cohort, whereas patients whom had a cytology verified ALNM pre-NACT and an axillary dissection performed $(N=114)$ defined a post-NACT cohort. AUS characteristics were prospectively collected pre- and post-NACT. The diagnostic accuracy of AUS was evaluated and stratified by histological subtype and body mass index (BMI). Predictors of axillary-pCR were analyzed, including MD, using simple and multivariable logistic regression models.

Results AUS demonstrated superior performance for prediction of ALNM pre-NACT in comparison to post-NACT, as reflected by the positive predictive value (PPV) 0.94 (95\% CI 0.89-0.97) and PPV 0.76 (95\% CI 0.62-0.87), respectively. We found no difference in AUS performance according to neither BMI nor histological subtype. Independent predictors of axillary-pCR were: premenopausal status, ER-negativity, HER2-overexpression, and high MD.

Conclusion Baseline AUS could, to a large extent, identify ALNM; however, post-NACT, AUS was insufficient to determine remaining ALNM. Thus, our results support the surgical staging of the axilla post-NACT. Baseline tumor biomarkers and patient characteristics were predictive of axillary-pCR. Larger, multicenter studies are needed to evaluate the performance of AUS post-NACT.
\end{abstract}

Keywords Breast cancer $\cdot$ Neoadjuvant chemotherapy $\cdot$ Imaging $\cdot$ Ultrasound $\cdot$ Axillary lymph nodes

\section{Abbreviations}

NACT Neoadjuvant chemotherapy

$\mathrm{BC}$

Breast cancer

Signe Borgquist and Lisa Rydén shared senior authorship.

Ida Skarping

ida.skarping@med.lu.se

1 Division of Oncology and Pathology, Department of Clinical Sciences, Lund University, Lund, Sweden

2 Department of Clinical Physiology and Nuclear Medicine, Skåne University Hospital, Lund, Sweden

3 Medical Radiation Physics, Department of Translational Medicine, Skåne University Hospital, Lund University, Malmö, Sweden

4 Diagnostic Radiology, Department of Translational Medicine, Department of Imaging and Functional
ALN Axillary lymph nodes

pCR Pathological complete response

ALND Axillary lymph node dissection

SLNB Sentinel lymph node biopsy

FNR False-negative rate

Medicine, Skåne University Hospital, Lund University, Lund and Malmö, Sweden

5 Department of Oncology, Aarhus University Hospital, Aarhus, Denmark

6 Division of Surgery, Department of Clinical Sciences, Lund University, Lund, Sweden

7 Department of Surgery, Skåne University Hospital, Lund, Sweden

8 Aarhus University, Aarhus, Denmark 


\begin{tabular}{|c|c|}
\hline SLN & Sentinel node \\
\hline AUS & Axillary ultrasound \\
\hline MRI & Magnetic resonance imaging \\
\hline FDG-PET/CT & $\begin{array}{l}{ }^{18} \text { F-fluorodeoxyglucose positron emission } \\
\text { tomography/computed tomography }\end{array}$ \\
\hline FNAC & Fine needle aspiration cytology \\
\hline ALNM & Axillary lymph nodes metastases \\
\hline HER2 & $\begin{array}{l}\text { Human epidermal growth factor receptor } \\
2\end{array}$ \\
\hline BI-RADS & $\begin{array}{l}\text { Breast Imaging-Reporting and Data } \\
\text { System }\end{array}$ \\
\hline $\mathrm{rCR}$ & Radiological complete response \\
\hline PPV & Positive predictive value \\
\hline NPV & Negative predictive value \\
\hline CI & Confidence interval \\
\hline BMI & Body mass index \\
\hline ER & Estrogen receptor \\
\hline
\end{tabular}

\section{Introduction}

Neoadjuvant chemotherapy (NACT) is the recommended treatment option for breast cancer (BC) patients with axillary lymph node (ALN)-positive disease [1]. The accomplishment of pathological complete response (pCR) following NACT, preferably including response in the breast and the axilla, is associated with improved prognosis [2, 3]. To avoid surgical over-treatment of the axilla, that is abstaining from axillary lymph node dissection (ALND) for patients that subsequently are shown to have axillary-pCR, high-performing imaging and/or less invasive surgical staging procedures are needed [4-7]. The SENTINA trial [4] and the Z1071 trial [5] are both prospective multicenter studies. The SENTINA trial was designed to evaluate the timing of sentinel lymph node biopsy (SLNB) in the NACT setting and the objective of the Z1071 trial was to determine the false-negative rate (FNR) for sentinel node (SLN) surgery following chemotherapy in women initially presenting with biopsy-proven $\mathrm{cN} 1 \mathrm{BC}$. In both studies, the primary endpoint was FNR of SLNB after NACT in patients presenting with upfront $\mathrm{cN} 1$ disease. The SENTINA trial and the Z1071 trial showed a FNR of 14\% and $13 \%$, respectively, for SLNB performed post-NACT, thus higher than the predefined threshold of $10 \%$. The SLN FNR was not different based on axillary ultrasound (AUS) results; however, using a strategy where only patients with normal AUS undergo SLN surgery reduced the FNR in patients with $\geq$ two SLNs removed included in the Z1071 trial from 12.6 to $9.8 \%$ when preoperative AUS results are considered as part of SLN surgery [8].

AUS is often the first-hand choice for axillary imaging, while more advanced methods for instance magnetic resonance imaging (MRI) and ${ }^{18} \mathrm{~F}$-fluorodeoxyglucose positron emission tomography/computed tomography (FDG-PET/
CT) are seldom routinely used [9]. At the time point of BC diagnosis, abnormal baseline AUS is routinely followed by ultrasound-guided fine needle aspiration cytology (FNAC); a quick minimally invasive method of axillary staging. FNACverified ALNM obviates SLNB, allowing the patient to proceed directly to ALND or, as for the patients in the present study, to NACT followed by ALND [10, 11]. However, current guidelines recommend that SLNB can safely be performed post-NACT for upfront $\mathrm{c} / \mathrm{pN}+$ patients and used as a discriminator for ALND [12, 13].

From an axillary surgery perspective, correct prediction of axillary-pCR is of utmost interest to enable abstaining from ALND. Identification of predictive imaging biomarkers of axillary-pCR is therefore important. In addition to breast tumor and ALN characteristics, mammographic density (MD) and its association with axillary-pCR, is investigated in this study. The timing of SLNB for BC patients treated with NACT is debated, and current guidelines [11, 12] recommend SLNB performed post-NACT and, in case of benign findings, omission of ALND $[4,5]$.

In this study, we report results from a well-characterized prospective cohort with AUS performed pre- and postNACT and detailed pathology data on ALN-metastases (ALNM) from both baseline (pre-NACT) and post-NACT (breast surgery and ALND). In addition, prospectively assessed mammographic density (qualitatively and quantitatively) at the corresponding time points were retrieved. While a large number of studies have investigated the performance of AUS pre-NACT [14, 15], only a few studies have investigated the performance of AUS post-NACT [6, $8,16-19]$ and not all report test performance data $[8,17]$. We investigated the test performing measures in terms of correctly identifying ALNM of AUS pre-NACT and, most importantly, post-NACT. Since overweight and a lobular BC subtype could be associated with inferior accuracy of AUS [20-22], stratification according to these parameters were performed. We also aimed to investigate the association between AUS parameters, as well as patient and tumor characteristics, respectively, as predictors of axillary-pCR.

\section{Methods}

The NeoDense-study cohort, a part of the SCAN-B study [23] (Clinical Trials ID NCT02306096), is a prospective cohort of BC patients receiving NACT during 2014-2019 at two sites within Skåne University Hospital, Sweden as previously described [24]. At diagnosis, patients eligible for NACT were included in the study following written consent $(N=207)$, of whom five patients were excluded due to ineligibility (Fig. 1) [25]. All patients with $\mathrm{c} / \mathrm{pN}+$ pre-NACT were subject to ALND according to clinical routine and the 
Fig. 1 Flowchart

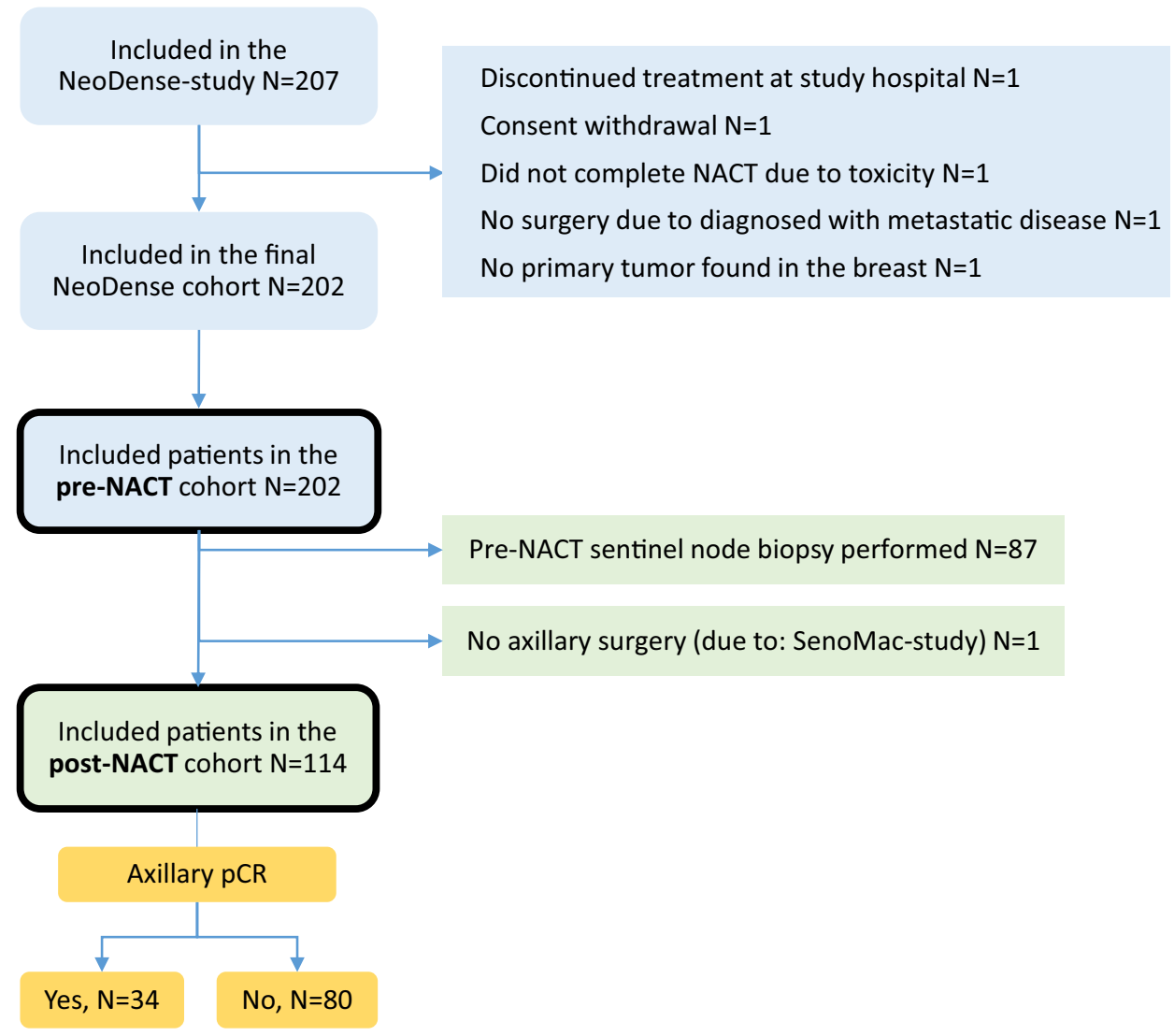

Swedish National Guidelines at the time of study inclusion (Supplementary Material 1).

\section{Pre-NACT cohort}

For the assessment of AUS at the pre-NACT time point, the whole NeoDense-cohort was used $(N=202)$, in order to include both baseline AUS abnormal and normal assessments. SLNB was performed pre-NACT for patients with clinically and AUS node-negative disease $(\mathrm{cNO})(N=87)$. Hence, the pathological diagnosis of ALN at baseline were based on assessment of SLNs in 87 patients and FNAC in 115 in patients with cytology verified ALNM (Fig. 1). SLNB was performed prior to study inclusion in 78 patients, and for these patients, the axillary evaluation of the preceding diagnostic AUS was used in the statistical analyses of the pre-NACT cohort.

\section{Post-NACT cohort}

From the 202 patients enrolled in the NeoDense study, we report on 114 (56\%) who had a cytology verified ALNM at baseline and had an ALND performed post-NACT. At the post-NACT time point, patients having pre-NACT SLNB-performed $(N=87)$ were excluded and $N=1$ patient was excluded due to being part of the SenoMac-study
(Clinical Trials ID NCT02240472) and thus no ALND was performed.

The term "axillary-pCR" commonly used in the literature, meaning no remaining invasive cancer in the axilla following NACT, is in this study only used for patients with FNAC-verified metastases at baseline; the term axillary-pCR was only applicable to patients with no previous surgical removal of ALN (due to SLNB).

\section{Clinical data}

Referring to the pre-NACT cohort $(N=202)$ : a total of $N=196$ (97\%) patients received standard chemotherapy regimen $[3 \times$ fluorouracil, epirubicin and cyclophosphamide (FEC), or epirubicin and cyclophosphamide (EC) $+3 \times$ docetaxel (or equivalent series of paclitaxel)], or in the reversed order. In the case of human epidermal growth factor receptor 2 (HER2) overexpression BC $(N=49)$, HER2-blockade was added $[N=46$, whereof $94 \%$ received double HER2-blockade (trastuzumab and pertuzumab), and the remaining three patients received single trastuzumab]. Data on clinical and pathological parameters were gathered from study-specific forms, medical charts, and clinical pathology reports. 


\section{Pathology evaluation}

FNAC of suspicious ALN was performed pre-NACT according to clinical routine by breast radiologists prior to study inclusion. Standard procedure included aspiration with 22 Gauge needle $(0.7 \mathrm{~mm} \times 50.0 \mathrm{~mm})$. All pathological interpretation was performed according to clinical routine at the pathology department by board certified cytopathologists. pCR was defined as no residual invasive cancer foci in the breast and axilla (ypT0/is ypN0), in accordance with current guidelines [26].

\section{Imaging}

Each patient had imaging examinations performed of the breast (mammography and ultrasound and parts of the cohort also breast tomosynthesis) and the axilla (AUS) at three time points: pre-NACT, after two series of NACT (during NACT), and post-NACT; the timing was mirroring clinical routine, and a detailed timeline of the cohort is already published [24]. Ultrasound assessment of ALNs was performed by experienced breast radiologists (specially trained and working at a breast imaging center $N=13$ ) and were considered abnormal or normal by evaluating the following criteria: nodal size, cortical thickening, hilar effacement, echogenicity, and shape [27]. No study-specific criteria for abnormal ALN was used; the assessment of normal/abnormal was at the discretion of the evaluating radiologist. Size, shape, cortex thickness above 3-4 mm, hilar effacement, and echogenicity were in a combined overall assessment used in the clinic by the radiologist to discriminate between normal/abnormal nodes. At the time of study inclusion, Breast ImagingReporting and Data System (BI-RADS) for AUS categorization was not used. The radiologists prospectively filled in study-specific forms at the time of AUS examinations including number of abnormal ALNs and their size(s) [28, 29] and echogenicity. For the post-NACT cohort, the number of valid long and short-axis measurement, respectively, for the abnormal AUS only at the different time points are presented in Table 2. The details of the ultrasound machines used are presented in Supplementary Material 2. Axillary radiological complete response (rCR) was defined as no abnormal findings (i.e., findings indicating malignancy) by AUS. Mammographic density was assessed both qualitatively by radiologists according to BI-RADS [30], and quantitatively with the automated software Volpara ${ }^{\mathrm{TM}}$ (version 1.5.4.0, Volpara Solutions Limited, Wellington, New Zealand) [31]. The breast tumor was marked with a radiopaque clip prior to NACT according to clinical routine, while no marking was performed of abnormal ALNs.

\section{Statistics}

We summarized cohort baseline characteristics, including pathology results from the breast and axilla. We calculated descriptive statistics according to axillary-pCR for ultrasound features of the breast tumor and ALN at three time points (baseline, during NACT, and post-NACT).

Test performance: For pre- and post-NACT cohort, we used axillary node-stage by AUS as a test for axillary nodestage by pathology/cytology, both at baseline $(N=202)$ and post-NACT (patients having FNAC-verified ALNM at baseline as well as ALND performed, $N=114$ ). We estimated test performance measures; sensitivity, specificity, positive, and negative predictive value (PPV and NPV) with 95\% confidence interval (CI) for the AUS-pathology association at baseline and post-NACT. Subgroup analyses were performed according to body constitution and histopathological subtype.

\section{Prediction models of axillary-pCR: post-NACT cohort}

We furthermore used simple logistic regression to assess whether baseline patient [age, body mass index (BMI), menopausal status], tumor characteristics [estrogen receptor (ER), HER2, Ki67], histopathological subtype, and imaging characteristics (tumor response, MD, and the number of abnormal ALN by AUS) were associated with axillarypCR (note that absence of ALNM is considered as outcome). In these models, axillary-pCR was the dependent variable, whereas the individual characteristic was included as an independent variable. To establish the independent association of these characteristics and axillary-pCR, we also conducted a fully adjusted multivariable logistic model. The independent variables in this axillary-pCR-model were deduced from simple and multivariable logistic regression models of different AUS parameters of abnormal ALN (number, long-axis, long/short-axis ratio), ultrasound breast tumor parameters (size and response), and MD at three different time points and their association with axillary-pCR. Each multivariable model included the baseline covariates from the previous model: model 1 , age; model 2 , model $1+$ BMI and menopausal status; and, model 3, model 2 + ER-status + HER2-status + Ki67 (all from core biopsies of the breast tumor at baseline).

\section{Statistical software}

For the test performance measures calculations, MedCalc Statistical Software version 19.2.6 (MedCalc Software Ltd, Ostend, Belgium; https://www.medcalc.org; 2020) was used. Otherwise, IBM SPSS Statistics for Windows, version 26 (IBM Corp., Armonk, N.Y., USA) was used. 


\section{Results}

\section{Descriptive results}

The baseline characteristics of the pre- and post-NACT cohort are displayed in Table 1. Invasive ductal carcinoma was the most common histopathological subtype in both the pre- and post-NACT cohort (165 of 202 (82\%) and 97 of 114 (85\%), respectively), followed by invasive lobular carcinoma (16 of 202 (8\%) and 9 of 114 (8\%), respectively). Regarding MD, most patients had intermediate MD (BI-RADS b or c combined accounted for 165 of $202(82 \%)$ and 91 of 114 $(80 \%)$ of the pre- and post-NACT cohort, respectively). The axillary-pCR rate was $30 \%$ (34 of 114).

The number and proportion of abnormal ALNs by AUS decreased during NACT in both the axillary-pCR and nonaxillary-pCR group (Table 2). Post-NACT, the proportion of normal ALN-status by AUS was 77\% (26 of 34) in the axillary-pCR group and 61\% (49 of 80) in non-axillary-pCR group. Of the 78 patients with SLNB prior to inclusion, $83 \%$ $(N=65)$ had no abnormal findings by AUS.

\section{Test performance}

Test performance measures of AUS pre- and post-NACT were stratified according to BMI and histological subtype, are presented in Fig. 2. Pre-NACT, a total of 123 of 202 (61\%) met abnormal AUS criteria (according to the expertise judgment by the radiologist), the corresponding number post-NACT was 38 of 114 (33\%). AUS showed better performance in terms of identifying ALNM pre-NACT $(N=202)$ as reflected by the PPV of 0.94 (95\% CI $0.89-0.97)$ and sensitivity of 0.81 (95\% CI $0.74-0.87)$. The performance of AUS was inferior post-NACT $(N=114)$; PPV 0.76 (95\% CI $0.62-0.87$ ) and sensitivity 0.35 (95\% CI $0.24-0.47)$. Stratified analyses according to BMI and histological subtype preand post-NACT showed no differences (Fig. 2).

\section{Prediction models of axillary-pCR: post-NACT cohort}

Baseline characteristics positively associated with accomplishing axillary-pCR in the simple and multivariable logistic regression analysis $(N=114)$ were: premenopausal status (OR 0.08 95\% CI 0.01-0.82), ER-negativity (OR 9.05 95\% CI 2.09-39.14), HER2-overexpression (OR 6.18 95\% CI 1.62-23.56), and mammographic dense breasts (OR 6.98 95\%CI 1.54-31.62) (Table 3). Tumor response as assessed with ultrasound (decrease $\geq 30 \%$ in largest diameter) between baseline and "during NACT" showed association with axillary-pCR in the unadjusted model (OR $2.6095 \% \mathrm{CI}$ 1.11-6.07); however, this association was not retained in the multivariable model (OR 1.48 95\% CI 0.43-5.08). The fully adjusted multivariable model including the 114 patients (adjusting for age, BMI, menopausal status, ER, HER2, and Ki67) is displayed in Supplementary Material 3, showing that the odds ratio for accomplishing axillary-pCR increased with the decreasing number of abnormal ALNs on AUS during (OR 0.46 95\% CI 0.25-0.83) and post-NACT (OR 0.58 95\%CI 0.30-1.10) (Supplementary Material 3).

\section{Discussion}

For BC patients receiving NACT, reliable imaging is needed both at baseline, in the initial staging-situation for wellgrounded systemic treatment decisions, as well as postNACT to optimize surgical treatment decisions. It is important to evaluate the performance of AUS, as has been studied multiple times before at the initial staging (pre-NACT or pre-primary BC surgery) $[14,15]$, but reported in a few previous studies post-NACT $[6,16,18,19]$. We present results of a well-characterized prospective cohort with extensive pathology data (complete data cytology proven ALNM at baseline and ALND post-NACT) and a detailed study protocol with sequential imaging (pre, during, and postNACT). Adding information to previously published studies, we present clinically valuable performance measures of AUS post-NACT. In addition to the many studies presenting nomograms (predominantly baseline data) for prediction of axillary-pCR [17, 32-38], this study presents novel findings of the association between MD and axillary-pCR.

\section{Test performance}

\section{AUS pre-NACT}

Our results show that baseline AUS could, to a large extent, correctly identify ALNM; the sensitivity, specificity, and PPV were satisfactory. However, a NPV of 0.65 (95\% CI 0.57-0.73) shows that pre-NACT AUS has limitations to correctly identify metastasis of any size in the axilla. This finding supports current guidelines that patients with clinically and AUS normal axilla at baseline can be staged by SLNB post-NACT without missing important information. The literature shows diverse sensitivity and specificity for the diagnosis of ALNM at baseline with AUS, ranging from 49 to $87 \%$ and 53 to $97 \%$, respectively $[14,15]$. This variety might partly be explained by the lacking consensus for imaging characteristics or scoring systems for abnormal ALN by AUS [15] and that ultrasound is a modality that has high intra- and inter-observer variability [39]. 
Table 1 Pre- and post-NACT cohort: patient, tumor, and axillary characteristics preNACT, and pathological/ radiological ALN-status postNACT

\begin{tabular}{|c|c|c|}
\hline & $\begin{array}{l}\text { Pre-NACT cohort } \\
(N=202)\end{array}$ & $\begin{array}{l}\text { Post-NACT } \\
\text { cohort } \\
(N=114)\end{array}$ \\
\hline \multicolumn{3}{|l|}{ Pre-NACT } \\
\hline Age at diagnosis, median (IQR) & $53(45-62)$ & $54(45-63)$ \\
\hline BMI, median (IQR) & $26(22-29)$ & $26(23-29)$ \\
\hline \multicolumn{3}{|l|}{ Menopausal status, $N(\%)$} \\
\hline Premenopausal & $96(47.5)$ & $51(44.7)$ \\
\hline Postmenopausal & $106(52.5)$ & $63(55.3)$ \\
\hline Tumor size as assessed by mammography, median (IQR) & $30(20-40)$ & $27(19-35)$ \\
\hline No detectable tumor, $N(\%)$ & $11(5.4)$ & $8(7.0)$ \\
\hline Tumor size not assessable, $N(\%)$ & $10(5.0)$ & $7(6.1)$ \\
\hline Test not performed, $N(\%)$ & $1(0.5)$ & $0(0)$ \\
\hline Tumor size as assessed by ultrasound, median (IQR) & $28(19-35)$ & $24(17-34)$ \\
\hline No detectable tumor, $N(\%)$ & $2(1.0)$ & $1(0.9)$ \\
\hline Tumorsize not assessable, $N(\%)$ & $5(2.5)$ & $2(1.8)$ \\
\hline \multicolumn{3}{|l|}{ Estrogen receptor status, $N(\%)$} \\
\hline Positive $(\geq 10 \%)$ & $121(59.9)$ & $83(72.8)$ \\
\hline Negative $(<10 \%)$ & $81(40.1)$ & $31(27.2)$ \\
\hline \multicolumn{3}{|l|}{ Progesterone receptor status, $N(\%)$} \\
\hline Positive $(\geq 10 \%)$ & $103(51.0)$ & $70(61.4)$ \\
\hline Negative $(<10 \%)$ & $98(48.5)$ & $43(37.7$ \\
\hline Missing & $1(0.5)$ & $1(0.9)$ \\
\hline \multicolumn{3}{|l|}{ HER2 receptor status ${ }^{\mathrm{a}}, N(\%)$} \\
\hline Positive & $49(24.3)$ & $24(21.1)$ \\
\hline Negative & $153(75.7)$ & $90(78.9)$ \\
\hline \multicolumn{3}{|l|}{$\mathrm{Ki} 67^{\mathrm{b}}, N(\%)$} \\
\hline Low & $11(5.4)$ & $87.0)$ \\
\hline Intermediate & $30(14.9)$ & $20(17.5)$ \\
\hline High & 159 (78.7) & $85(74.6)$ \\
\hline Missing & $2(1.0)$ & $1(0.9$ \\
\hline \multicolumn{3}{|l|}{ Histopathological subtype, $N(\%)$} \\
\hline Ductal & $165(81.7)$ & $97(85.1)$ \\
\hline Lobular & $16(7.9)$ & $9(7.9)$ \\
\hline Other & $12(5.9)$ & $4(3.5)$ \\
\hline Missing & $9(4.5)$ & $4(3.5)$ \\
\hline \multicolumn{3}{|l|}{ Mammographic density } \\
\hline VBD\%, median (IQR) & $11.5(7.6-18.3)$ & $10.4(6.9-17.2)$ \\
\hline Missing, $N(\%)$ & $9(4.5)$ & $4(3.5)$ \\
\hline \multicolumn{3}{|l|}{ BI-RADS, N (\%) } \\
\hline A & $9(4.5)$ & $6(5.3)$ \\
\hline $\mathrm{B}$ & $74(36.6)$ & $43(37.7)$ \\
\hline $\mathrm{C}$ & $91(45.0)$ & $48(42.1)$ \\
\hline $\mathrm{D}$ & $27(13.4)$ & $17(14.9)$ \\
\hline Missing & $1(0.5)$ & $1(0.5)$ \\
\hline \multicolumn{3}{|l|}{ Abnormal ALN by AUS, $N(\%)$} \\
\hline Yes & $123(60.9)$ & $109(95.6)$ \\
\hline No & $79(39.1)$ & $5(4.4)$ \\
\hline \multicolumn{3}{|l|}{ Post-NACT } \\
\hline \multicolumn{3}{|l|}{ Axillary-pCR, $N(\%)$} \\
\hline Yes & N/A & $34(29.8)$ \\
\hline No & N/A & $80(70.2)$ \\
\hline
\end{tabular}


Table 1 (continued)

\begin{tabular}{lll}
\hline & $\begin{array}{l}\text { Pre-NACT cohort } \\
(N=202)\end{array}$ & $\begin{array}{l}\text { Post-NACT } \\
\text { cohort } \\
(N=114)\end{array}$ \\
\hline Abnormal ALN by AUS, $N(\%)$ & $41(20.3)$ & $38(33.3)$ \\
Yes & $155(76.7)$ & $75(65.8)$ \\
No & $6(3.0)$ & $1(0.9)$ \\
Missing & $6(0)$ & \\
\hline
\end{tabular}

$B M I$ body mass index, $H E R 2$ human epidermal growth factor receptor 2, $V B D \%$ volumetric breast density percentage, $B I-R A D S$ Breast Imaging-Reporting and Data System, $A L N$ axillary lymph nodes, $A U S$ axillary ultrasound, $p C R$ pathological complete response

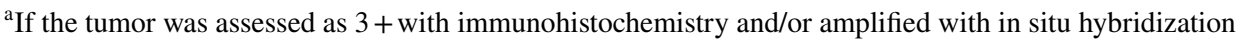

${ }^{\mathrm{b}}$ Tumors were considered as low, intermediate, or highly proliferative according to laboratory specific cutoffs (site 1: low 0-20\%; intermediate 21-30\%; high 31-100\%, site 2: low 0-14\%; intermediate 15-24\%; high $25-100 \%$ ) for proportion of cells staining positive for Ki67

\section{AUS post-NACT}

In more recent years, classification systems of ultrasound evaluation of ALN post-NACT have been presented to determine important ALN characteristics to consider postNACT [40]. Importantly, in our study, the sensitivity of AUS post-NACT (identifying ALNM) was considerably lower in comparison to pre-NACT, but the specificity and PPV were acceptable. Thus, AUS could not identify all (subsequent pathology-verified) ALNM. Previous studies have shown sensitivity and specificity rates for AUS (of identification of ALNM) post-NACT of 50-60\% and 60-77\%, respectively $[6,18]$. In the SN FNAC study [19], the PPV and NPV of AUS post-NACT were slightly higher than in the present study (PPV $81 \%$ and NPV 48\% in SN FNAC-study in comparison to PPV $76 \%$ and NPV $38 \%$ in our study). These results are to be expected since ultrasound is reflecting a macroscopic feature in contrast to the remaining microscopic findings in the pathology specimen. The study samples are similar ( $\mathrm{N}$ ranging from $139-157[6,18,19])$ to ours except for the large cohort in the SENTINA trial [16].

\section{Other modalities}

Several strategies in improving the diagnostic performance of axillary staging by imaging have been proposed. In our study, we used a limited number of easily accessible, clinically established AUS parameters [41]. Also, other imaging modalities for axillary staging pre- and post-NACT must be mentioned. In the primary stagingsetting, a review by Marino et al. showed pooled estimates of the sensitivity of $75-80 \%$ and $59-69 \%$ for MRI and FDG-PET/CT, respectively, and the corresponding estimates for specificity were $89-91 \%$ and $90-95 \%$ for MRI and FDG-PET/CT, respectively [15]. When examining the axilla post-NACT, a study using MRI $(N=65)$ has shown a PPV of $67 \%$ and a NPV of $66 \%$ of biopsy-proven ALNM
pre-NACT in terms of predicting axillary-pCR [42]; the corresponding numbers for AUS in our study was PPV $38 \%$ and NPV $76 \%$. A comparative study between AUS, MRI, and FDG-PET/CT presented post-NACT sensitivity of axillary imaging in detecting ALNM to be $70 \%$ for AUS $(N=106), 61 \%$ for MRI $(N=88)$, and $63 \%$ for FDG-PET/CT $(N=32)$ [43]. Another study comparing different modalities' [ultrasound $(N=135)$, MRI $(N=136)$, and FDG-PET/CT $(N=99)$ ], and combinations thereof, test performance measures post-NACT, showed NPV ranging from 28 to $48 \%$, the latter from the combination of AUS and MRI [6]. In conclusion, studies of these advanced imaging modalities post-NACT have a limited number of study participants and a comprehensive overview of axillary imaging post-NACT, including AUS, is thus warranted.

\section{AUS, BMI, and histopathological subtype}

Since clinical axillary palpation might be more challenging in overweight/obese patients, AUS is of even greater importance for these patients. In overweight and obese patients AUS could be afflicted with inferior performance due to technical challenges and obesity-related ALN-alterations [44, 45]; however, studies of baseline AUS points toward no impediment [45]. To the best of our knowledge, not previously reported in the literature, we present results of test performance of AUS post-NACT in relation to BMI. In adherence to studies of AUS at baseline, we found no difference in AUS performance according to BMI at either time point. Previous studies [20, 21] have indicated inferior accuracy of AUS in lobular cancer. In the present study, we found inconclusive results in terms of AUS performance at each time points due to insufficient number of patients in the lobular histopathology groups $(N=14$ pre-NACT and $N=9$ post-NACT $)$. 


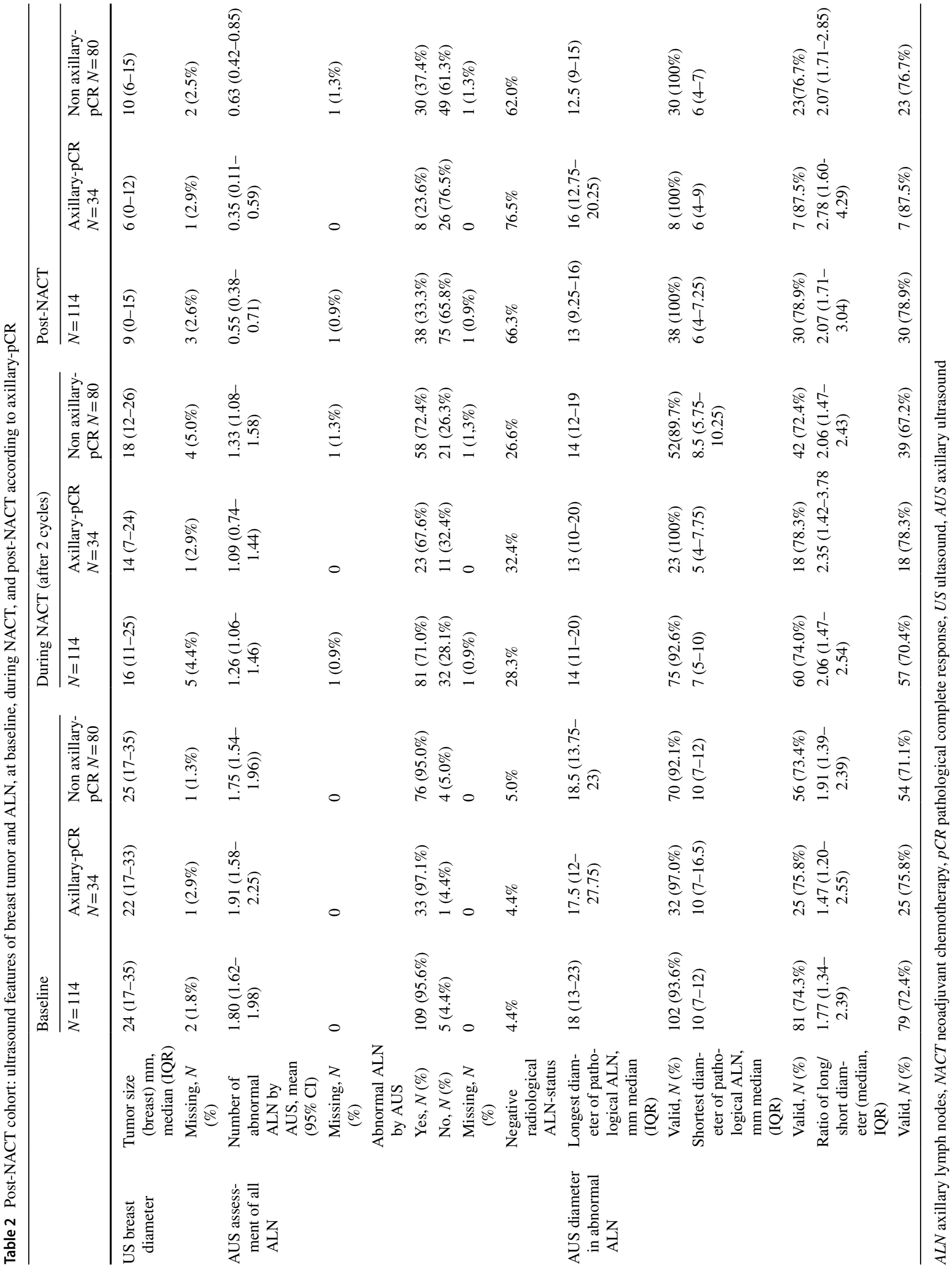


Table 3 Post-NACT cohort: simple and multivariable logistic regression analysis of baseline tumor, patients characteristics, and imaging characteristics during/post-NACT as predictors of axillary-pCR following NACT

\begin{tabular}{|c|c|c|c|c|c|c|}
\hline \multirow[t]{2}{*}{ Variables } & \multicolumn{2}{|c|}{ Simple logistic regression } & \multirow[b]{2}{*}{$N$} & \multicolumn{2}{|c|}{$\begin{array}{l}\text { Multivariable* logistic regres- } \\
\text { sion }\end{array}$} & \multirow[b]{2}{*}{$N$} \\
\hline & OR $(95 \% \mathrm{CI})$ & $p$ value & & OR $(95 \% \mathrm{CI})$ & $p$ value & \\
\hline \multicolumn{7}{|l|}{ Patient and tumor characteristics (baseline) } \\
\hline Age (continuous) & $0.97(0.94-1.01)$ & 0.11 & 114 & $0.92(0.84-1.01)$ & 0.08 & 94 \\
\hline BMI (continuous) & $0.97(0.89-1.07)$ & 0.55 & 114 & $0.94(0.82-1.09)$ & 0.42 & 94 \\
\hline Postmenopausal & $1.29(0.58-2.87)$ & 0.54 & 114 & $0.08(0.01-0.82)$ & 0.03 & 94 \\
\hline ER-negativity & $6.53(2.65-16.07)$ & $<0.01$ & 114 & $9.05(2.09-39.14)$ & $<0.01$ & 94 \\
\hline HER2-overexpression & $5.53(2.14-14.25)$ & $<0.01$ & 114 & $6.18(1.62-23.56)$ & $<0.01$ & 94 \\
\hline Ki67 (continuous) & $1.03(1.01-1.05)$ & $<0.01$ & 113 & $1.02(0.99-1.05)$ & 0.31 & 94 \\
\hline Histopathological subtype-ductal (ref) & & & 97 & & & 86 \\
\hline Lobular & $0.64(0.13-3.26)$ & 0.59 & 9 & $0.72(0.05-10.34)$ & 0.81 & 6 \\
\hline Other & $0.74(0.07-7.45)$ & 0.80 & 4 & $4.03(0.17-97.16)$ & 0.39 & 2 \\
\hline \multicolumn{7}{|l|}{ Imaging characteristics } \\
\hline Tumor response during NACT (decrease $\geq 30 \%$, yes/no) & $2.60(1.11-6.07)$ & 0.03 & 107 & $1.48(0.43-5.08)$ & 0.53 & 94 \\
\hline $\begin{array}{l}\text { Mammographic density: BI-RADS dichotomized post- } \\
\text { NACT (ref A/B"non-dense") }\end{array}$ & & & 48 & & & 42 \\
\hline C/D ("dense") & $2.85(1.16-6.97)$ & 0.02 & 58 & $6.98(1.54-31.62)$ & 0.01 & 52 \\
\hline Number of abnormal ALN post-NACT by AUS & $0.66(0.39-1.13)$ & 0.13 & 113 & $0.58(0.28-1.24)$ & 0.16 & 94 \\
\hline
\end{tabular}

$N A C T$ neoadjuvant chemotherapy, $p C R$ pathological complete response, $O R$ odds ratio, $C I$ confidence interval, $B M I$ body mass index, $E R$ estrogen receptor, HER2 human epidermal growth factor receptor 2, BI-RADS Breast Imaging-Reporting and Data System, $A L N$ axillary lymph nodes, $A U S$ axillary ultrasound

"adjusted for: age, BMI, menopausal status, ER, HER2, Ki67, histopathological subtype, number of positive ALN by ultrasound post-NACT, tumor size decrease (by ultrasound) $\geq 30 \%$ during NACT, and mammographic density (BI-RADS dichotomized post NACT)

\section{Prediction models of axillary-pCR: post-NACT cohort}

An important finding in our study is the results from our simple logistic regression analysis of patient and tumor characteristics: the associations between ER-negativity and HER2-overexpression, respectively, and axillary-pCR were more pronounced than for many of the imaging characteristics of the breast and the axilla. Our results are in line with previous studies presenting predictive models, recognizing the importance of pre-NACT tumor characteristics [17, $32-38,46]$. These studies have similar odds ratio of axillarypCR as in the present study, thus adding credibility to our results. Similar to the tumor in the breast, ALN response to NACT is dependent on BC tumor subtype [47].

\section{Mammographic density}

Mammographic density, reflecting the radiodense stroma and epithelium of the breast on a mammogram [48], is associated with increased risk of BC development [49], higher risk of recurrence [50], and possibly poorer response to treatment [51, 52], although inconsistent results have been presented [24]. BC tumors in mammographic dense breasts are often larger at diagnosis and have positive ALN [53], thus justifying exploring the association between MD and rate of axillary-pCR. We did not find any association between MD assessed with Volpara ${ }^{\mathrm{TM}}$ and the likelihood of accomplishing axillary-PCR. In contrast, the BI-RADS assessment showed that dense breasts (BI-RADS c/d) were associated with higher odds ratio of accomplishing axillarypCR in comparison to non-dense (BI-RADS a/b), an association more pronounced at the later time points. To the best of the authors' knowledge, no previous studies have addressed MD vs. axillary-pCR.

\section{Future perspective}

The timing of SLNB is under scientific and clinical debate [4]. In $40-65 \%$ of patients with positive SLN at baseline, the $\operatorname{SLN}(\mathrm{s})$ is expected to be the only positive ALN, meaning that many of these patients do not have ALNM left in the axilla during and post-NACT $[54,55]$. SLNB offers a reliable staging at baseline [56]; in patients with a benign SLNB pre-NACT, it is considered safe to omit further axillary surgery, conditionally not progressing during NACT [57]. Patients in our cohort were treated according to this clinical algorithm. Correspondingly, there is an ongoing discussion of alternative treatment strategies to ALND for upfront ALN-positive patients with axillary-rCR postNACT. To reduce the morbidity related to ALND [58], less 

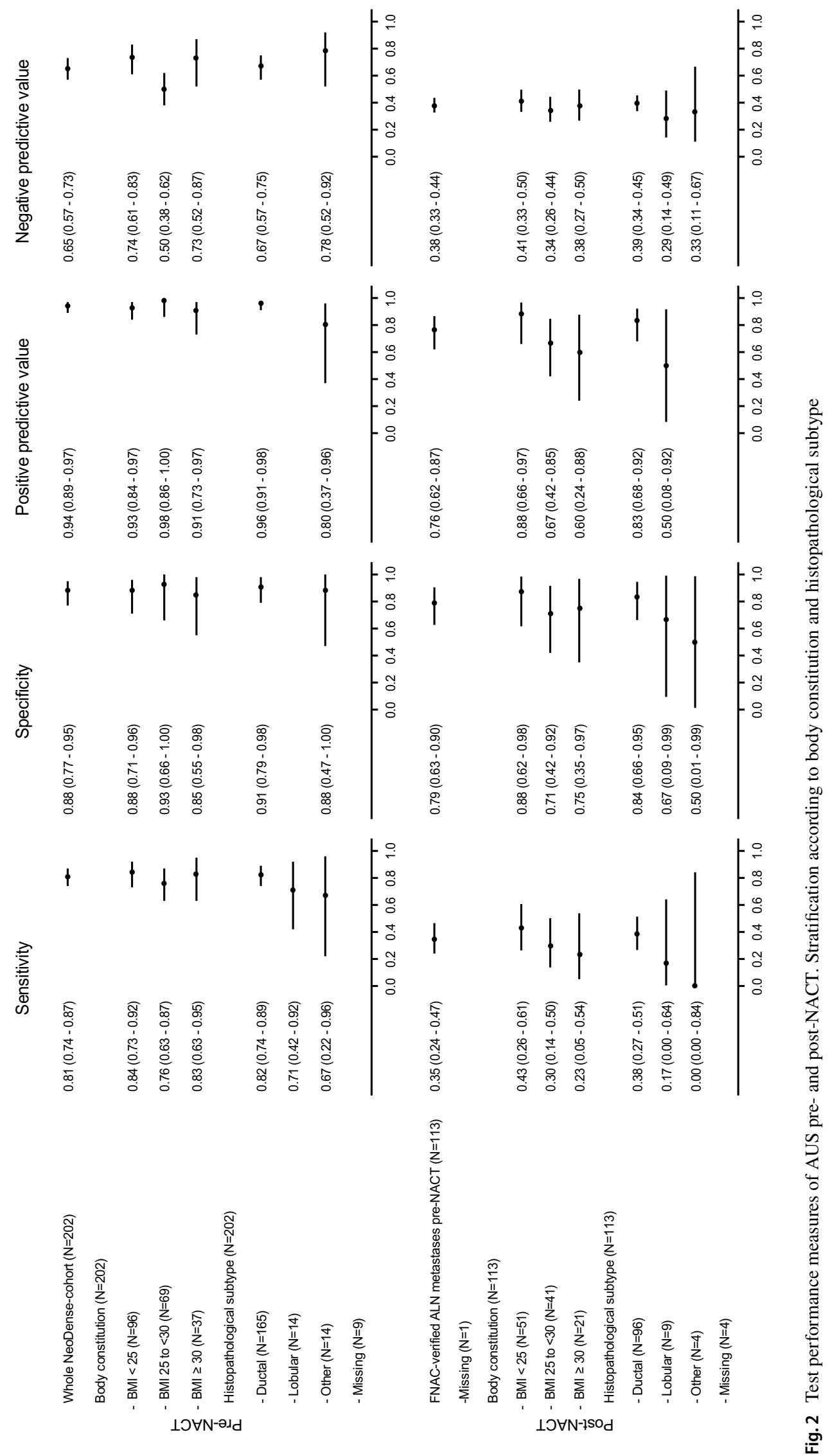
invasive procedures and treatment strategies are warranted. High demands are put on imaging, of which ultrasound is considered to be the preferred choice for axillary assessment [9], and used as a discriminator for patients eligible for SLNB [9]. Likewise, performed either pre-NACT, or as current guidelines recommend, post-NACT $[12,13]$; the test performance measures of SNLB must be high-level, most importantly with low FNR [59]. Our results indicate that AUS is a good predictor of ALNM at baseline, supporting abstaining from SLNB before NACT. However, AUS assessment post-NACT was not able to correctly diagnose remaining tumor deposits in patients with ALNM at the time of diagnosis.

\section{Strengths and limitations}

Our study has many strengths, including the prospective design with detailed data on patient, tumor, and breast characteristics at several time points. Since the guidelines are currently changing, recommending SLNB post-NACT, a similar study to our study and the SENTINA study [4] will be difficult to perform in the future. Possibly, access to breast MRI, could have been beneficial; however, axillaryMRI (provided a dedicated axillary protocol) has a minor role in axillary assessment [15]. Applicable to many imaging studies of the axilla, the lack of a standardized system of reporting findings (e.g., BI-RADS [30]) contributes to a wide variety of AUS test performance measures. To the best of the authors' knowledge, no guidelines exist on a national or European level regarding assessment of abnormal/normal ALN. Another shortcoming is the incomplete data on ALN short-axis measures (used in the RECIST criteria [60]). Consequently, only a limited number of patients had data on long/short axis ratio, an established measure mirroring the shape of the ALN (round or elongated) [41]. At baseline, according to clinical routine, FNAC was used to verify abnormal ALN by AUS. However, core biopsy to the ALN is considered to have higher diagnostic accuracy and is currently introduced [61]. Selection bias should be briefly addressed; although many patients had a positive ALN at baseline due to NACT being a preferred treatment option for patients with cytology/pathology-verified ALN at baseline, an abnormal ALN-status by AUS was not an inclusion criterion, and the selection bias should thus be minor.

\section{Conclusion}

Prior to NACT, AUS could, to a large extent, correctly identify abnormal ALN, supporting the omission of SLNB pre-NACT. In contrast, AUS alone is not sufficient to determine remaining ALNM post-NACT, whereas tumor biomarkers at baseline are predictive of axillary-pCR. We found no difference in AUS performance according to BMI at any time point. Larger multi-center studies are needed to evaluate the performance of AUS post-NACT. Investigation of other imaging modalities for treatment evaluation post-NACT is encouraged.

Supplementary Information The online version contains supplementary material available at https://doi.org/10.1007/s10549-021-06283-8.

Acknowledgements We express our gratitude to all the study participants. We acknowledge the outstanding work performed by research nurse Lina Zander and the valuable statistical input from Uffe HeideJørgensen. Lastly, we thank all the personnel at Unilabs Malmö and Helsingborg for their cooperation.

Author contributions IS assisted in the overall study design, created the study protocols, organized the enrollment of patients and the data collection, wrote the statistical plan, performed the statistical analyses, interpreted the data, solely drafted the manuscript and organized the revision of the manuscript. DF delivered technical aid during image gathering, interpreted the data, and revised the manuscript. UH substantially participated in discussions regarding the statistical plan, interpreted the data, and revised the manuscript. SZ partook in the over-all design of the study, critically interpreted the results, and contributed to the revision of the manuscript. SB was the principal contributor to the initial design of the NeoDense study, interpreted the data/results, and revised the manuscript. LR was co-initiator to this particular study, participated in the planning of the statistical analyses and in data interpretation, as well as intellectually revised the manuscript. All authors have read and approved the final manuscript.

Funding Open access funding provided by Lund University. This work was supported by grants from the Swedish Breast Cancer Group (BRO), The Erling Persson Family Foundation, and the Governmental Funding of Clinical Research within National Health Services (“ALF”). The funding resources had no role in the study design, data collection, analyses, data interpretation, writing of the manuscript, or the decision to submit the manuscript for publication.

Data availability The datasets used and/or analyzed during the current study are available from the corresponding author on reasonable request.

\section{Declarations}

Conflict of interest SZ has received speakers' fees and travel support from Siemens Healthcare AG, and consultancy fees from Collective Minds Radiology AB. SB has received speakers' fees from Pfizer, is a study-specific advisor Pfizer, and has received travel support from Roche. The other authors declare that they have no competing interests.

Ethical approval All procedures performed in studies involving human participants were in accordance with the ethical standards of the institutional and/or national research committee and with the 1964 Helsinki declaration and its later amendments or comparable ethical standards. The study was approved by the Regional Ethics Committee in Lund, Sweden (Official records number: 2014/13, 2014/521 and 2016/521).

Consent to participate Informed written consent was obtained from all participants included in the study.

Consent for publication Consent for publication was obtained from all participants included in the study. 
Open Access This article is licensed under a Creative Commons Attribution 4.0 International License, which permits use, sharing, adaptation, distribution and reproduction in any medium or format, as long as you give appropriate credit to the original author(s) and the source, provide a link to the Creative Commons licence, and indicate if changes were made. The images or other third party material in this article are included in the article's Creative Commons licence, unless indicated otherwise in a credit line to the material. If material is not included in the article's Creative Commons licence and your intended use is not permitted by statutory regulation or exceeds the permitted use, you will need to obtain permission directly from the copyright holder. To view a copy of this licence, visit http://creativecommons.org/licenses/by/4.0/.

\section{References}

1. Burstein HJ, Curigliano G, Loibl S, Dubsky P, Gnant M, Poortmans P, Colleoni M, Denkert C, Piccart-Gebhart M, Regan M et al (2019) Estimating the benefits of therapy for early-stage breast cancer: the St. Gallen International Consensus Guidelines for the primary therapy of early breast cancer 2019. Ann Oncol 30(10):1541-1557

2. von Minckwitz G, Untch M, Blohmer JU, Costa SD, Eidtmann H, Fasching PA, Gerber B, Eiermann W, Hilfrich J, Huober J et al (2012) Definition and impact of pathologic complete response on prognosis after neoadjuvant chemotherapy in various intrinsic breast cancer subtypes. J Clin Oncol 30(15):1796-1804

3. Cortazar P, Zhang L, Untch M, Mehta K, Costantino JP, Wolmark N, Bonnefoi H, Cameron D, Gianni L, Valagussa P et al (2014) Pathological complete response and long-term clinical benefit in breast cancer: the CTNeoBC pooled analysis. Lancet 384(9938):164-172

4. Kuehn T, Bauerfeind I, Fehm T, Fleige B, Hausschild M, Helms G, Lebeau A, Liedtke C, von Minckwitz G, Nekljudova V et al (2013) Sentinel-lymph-node biopsy in patients with breast cancer before and after neoadjuvant chemotherapy (SENTINA): a prospective, multicentre cohort study. Lancet Oncol 14(7):609-618

5. Boughey JC, Suman VJ, Mittendorf EA, Ahrendt GM, Wilke LG, Taback B, Leitch AM, Kuerer HM, Bowling M, Flippo-Morton TS et al (2013) Sentinel lymph node surgery after neoadjuvant chemotherapy in patients with node-positive breast cancer: the ACOSOG Z1071 (Alliance) clinical trial. JAMA 310(14):1455-1461

6. You S, Kang DK, Jung YS, An YS, Jeon GS, Kim TH (2015) Evaluation of lymph node status after neoadjuvant chemotherapy in breast cancer patients: comparison of diagnostic performance of ultrasound, MRI and (1)(8)F-FDG PET/CT. Br J Radiol 88(1052):20150143

7. Simons JM, van Nijnatten TJA, van der Pol CC, Luiten EJT, Koppert LB, Smidt ML (2019) Diagnostic accuracy of different surgical procedures for axillary staging after neoadjuvant systemic therapy in node-positive breast cancer: a systematic review and meta-analysis. Ann Surg 269(3):432-442

8. Boughey JC, Ballman KV, Hunt KK, McCall LM, Mittendorf EA, Ahrendt GM, Wilke LG, Le-Petross HT (2015) Axillary ultrasound after neoadjuvant chemotherapy and its impact on sentinel lymph node surgery: results from the American College of Surgeons Oncology Group Z1071 Trial (Alliance). J Clin Oncol 33(30):3386-3393

9. Expert Panel on Breast I, Slanetz PJ, Moy L, Baron P, diFlorio RM, Green ED, Heller SL, Holbrook AI, Lee SJ, Lewin AA et al (2017) ACR appropriateness criteria((R)) monitoring response to neoadjuvant systemic therapy for breast cancer. J Am Coll Radiol 14(11S):S462-S475
10. Ecanow JS, Abe H, Newstead GM, Ecanow DB, Jeske JM (2013) Axillary staging of breast cancer: what the radiologist should know. Radiographics 33(6): 1589-1612

11. Bröstcancer -Nationellt vårdprogram 2020, https://kunskapsba nken.cancercentrum.se/globalassets/cancerdiagnoser/brost/vardp rogram/nationellt-vardprogram-brostcancer.pdf. Accessed 30 Sept 2020

12. Liedtke C, Jackisch C, Thill M, Thomssen C, Muller V, Janni W, Committee AGOB (2018) AGO recommendations for the diagnosis and treatment of patients with early breast cancer: update 2018. Breast Care (Basel) 13(3):196-208

13. Cardoso F, Kyriakides S, Ohno S, Penault-Llorca F, Poortmans P, Rubio IT, Zackrisson S, Senkus E (2019) Early breast cancer: ESMO Clinical Practice Guidelines for diagnosis, treatment and follow-updagger. Ann Oncol 30(8):1194-1220

14. Alvarez S, Anorbe E, Alcorta P, Lopez F, Alonso I, Cortes J (2006) Role of sonography in the diagnosis of axillary lymph node metastases in breast cancer: a systematic review. Am J Roentgenol 186(5):1342-1348

15. Marino MA, Avendano D, Zapata P, Riedl CC, Pinker K (2020) Lymph node imaging in patients with primary breast cancer: concurrent diagnostic tools. Oncologist 25(2):e231-e242

16. Schwentner L, Helms G, Nekljudova V, Ataseven B, Bauerfeind I, Ditsch N, Fehm T, Fleige B, Hauschild M, Heil J et al (2017) Using ultrasound and palpation for predicting axillary lymph node status following neoadjuvant chemotherapy-results from the multi-center SENTINA trial. Breast 31:202-207

17. Kim HS, Shin MS, Kim CJ, Yoo SH, Yoo TK, Eom YH, Chae BJ, Song BJ (2017) Improved model for predicting axillary response to neoadjuvant chemotherapy in patients with clinically nodepositive breast cancer. J Breast Cancer 20(4):378-385

18. Ha SM, Cha JH, Kim HH, Shin HJ, Chae EY, Choi WJ (2017) Diagnostic performance of breast ultrasonography and MRI in the prediction of lymph node status after neoadjuvant chemotherapy for breast cancer. Acta Radiol 58(10):1198-1205

19. Boileau JF, Poirier B, Basik M, Holloway CM, Gaboury L, Sideris L, Meterissian S, Arnaout A, Brackstone M, McCready DR et al (2015) Sentinel node biopsy after neoadjuvant chemotherapy in biopsy-proven node-positive breast cancer: the SN FNAC study. J Clin Oncol 33(3):258-264

20. Morrow E, Lannigan A, Doughty J, Litherland J, Mansell J, Stallard S, Mallon E, Romics L (2018) Population-based study of the sensitivity of axillary ultrasound imaging in the preoperative staging of node-positive invasive lobular carcinoma of the breast. Br J Surg 105(8):987-995

21. Upadhyaya VS, Lim GH, Chan EYK, Fook-Chong SMC, Leong LCH (2020) Evaluating the preoperative breast cancer characteristics affecting the accuracy of axillary ultrasound staging. Breast J 26(2):162-167

22. Dihge L, Grabau DA, Rasmussen RW, Bendahl PO, Ryden L (2016) The accuracy of preoperative axillary nodal staging in primary breast cancer by ultrasound is modified by nodal metastatic load and tumor biology. Acta Oncol 55(8):976-982

23. Saal LH, Vallon-Christersson J, Hakkinen J, Hegardt C, Grabau D, Winter C, Brueffer C, Tang MH, Reutersward C, Schulz R et al (2015) The Sweden Cancerome Analysis Network - Breast (SCAN-B) Initiative: a large-scale multicenter infrastructure towards implementation of breast cancer genomic analyses in the clinical routine. Genome Med 7(1):20

24. Skarping I, Fornvik D, Heide-Jorgensen U, Sartor H, Hall P, Zackrisson S, Borgquist S (2020) Mammographic density changes during neoadjuvant breast cancer treatment: NeoDense, a prospective study in Sweden. Breast 53:33-41

25. Skarping I, Fornvik D, Heide-Jorgensen U, Ryden L, Zackrisson S, Borgquist S (2020) Neoadjuvant breast cancer treatment 
response; tumor size evaluation through different conventional imaging modalities in the NeoDense study. Acta Oncol 59:1-10

26. Bossuyt V, Provenzano E, Symmans WF, Boughey JC, Coles C, Curigliano G, Dixon JM, Esserman LJ, Fastner G, Kuehn T et al (2015) Recommendations for standardized pathological characterization of residual disease for neoadjuvant clinical trials of breast cancer by the BIG-NABCG collaboration. Ann Oncol 26(7):1280-1291

27. Krishnamurthy S, Sneige N, Bedi DG, Edieken BS, Fornage BD, Kuerer HM, Singletary SE, Hunt KK (2002) Role of ultrasoundguided fine-needle aspiration of indeterminate and suspicious axillary lymph nodes in the initial staging of breast carcinoma. Cancer 95(5):982-988

28. Dialani V, James DF, Slanetz PJ (2015) A practical approach to imaging the axilla. Insights Imaging 6(2):217-229

29. Park SH, Jeong YM, Cho SH, Jung HK, Kim SJ, Ryu HS (2014) Imaging findings of variable axillary mass and axillary lymphadenopathy. Ultrasound Med Biol 40(9):1934-1948

30. Sickles E, D'Orsi CJ, Bassett LW et al (2013) ACR BI-RADS® mammography. ACR BI-RADS $®$ atlas, breast imaging reporting and data system. American College of Radiology, Reston

31. Highnam R, Brady SM, Yaffe MJ, Karssemeijer N, Harvey J (2010) Robust breast composition measurement-VolparaTM. Springer Berlin Heidelberg, Berlin, Heidelberg, pp 342-349

32. Vila J, Mittendorf EA, Farante G, Bassett RL, Veronesi P, Galimberti V, Peradze N, Stauder MC, Chavez-MacGregor M, Litton JF et al (2016) Nomograms for predicting axillary response to neoadjuvant chemotherapy in clinically node-positive patients with breast cancer. Ann Surg Oncol 23(11):3501-3509

33. Schipper RJ, Moossdorff M, Nelemans PJ, Nieuwenhuijzen GA, de Vries B, Strobbe LJ, Roumen RM, van den Berkmortel F, TjanHeijnen VC, Beets-Tan RG et al (2014) A model to predict pathologic complete response of axillary lymph nodes to neoadjuvant chemo(immuno)therapy in patients with clinically node-positive breast cancer. Clin Breast Cancer 14(5):315-322

34. Murphy BL, Hoskin TL, Heins CDN, Habermann EB, Boughey JC (2017) Preoperative prediction of node-negative disease after neoadjuvant chemotherapy in patients presenting with node-negative or node-positive breast cancer. Ann Surg Oncol 24(9):2518-2525

35. Jin X, Jiang YZ, Chen S, Shao ZM, Di GH (2016) A nomogram for predicting the pathological response of axillary lymph node metastasis in breast cancer patients. Sci Rep 6:32585

36. Kantor O, Sipsy LM, Yao K, James TA (2018) A predictive model for axillary node pathologic complete response after neoadjuvant chemotherapy for breast cancer. Ann Surg Oncol 25(5):1304-1311

37. Kim JY, Park HS, Kim S, Ryu J, Park S, Kim SI (2015) Prognostic nomogram for prediction of axillary pathologic complete response after neoadjuvant chemotherapy in cytologically proven node-positive breast cancer. Medicine (Baltimore) 94(43):e1720

38. Ouldamer L, Chas M, Arbion F, Body G, Cirier J, Ballester M, Bendifallah S, Darai E (2018) Risk scoring system for predicting axillary response after neoadjuvant chemotherapy in initially node-positive women with breast cancer. Surg Oncol 27(2):158-165

39. Choi HY, Park M, Seo M, Song E, Shin SY, Sohn YM (2017) Preoperative axillary lymph node evaluation in breast cancer: current issues and literature review. Ultrasound Q 33(1):6-14

40. Le-Petross HT, McCall LM, Hunt KK, Mittendorf EA, Ahrendt GM, Wilke LG, Ballman KV, Boughey JC (2018) Axillary ultrasound identifies residual nodal disease after chemotherapy: results from the American College of Surgeons Oncology Group Z1071 Trial (Alliance). Am J Roentgenol 210(3):669-676

41. Ganeshalingam S, Koh DM (2009) Nodal staging. Cancer Imaging 9:104-111
42. Weber JJ, Jochelson MS, Eaton A, Zabor EC, Barrio AV, Gemignani ML, Pilewskie M, Van Zee KJ, Morrow M, El-Tamer M (2017) MRI and prediction of pathologic complete response in the breast and axilla after neoadjuvant chemotherapy for breast cancer. J Am Coll Surg 225(6):740-746

43. Hieken TJ, Boughey JC, Jones KN, Shah SS, Glazebrook KN (2013) Imaging response and residual metastatic axillary lymph node disease after neoadjuvant chemotherapy for primary breast cancer. Ann Surg Oncol 20(10):3199-3204

44. Giovagnorio F, Drudi FM, Fanelli G, Flecca D, Francioso A (2005) Fatty changes as a misleading factor in the evaluation with ultrasound of superficial lymph nodes. Ultrasound Med Biol 31(8): 1017-1022

45. Shah AR, Glazebrook KN, Boughey JC, Hoskin TL, Shah SS, Bergquist JR, Dupont SC, Hieken TJ (2014) Does BMI affect the accuracy of preoperative axillary ultrasound in breast cancer patients? Ann Surg Oncol 21(10):3278-3283

46. Kim R, Chang JM, Lee HB, Lee SH, Kim SY, Kim ES, Cho N, Moon WK (2019) Predicting axillary response to neoadjuvant chemotherapy: breast MRI and US in patients with node-positive breast cancer. Radiology 293(1):49-57

47. Boughey JC, McCall LM, Ballman KV, Mittendorf EA, Ahrendt GM, Wilke LG, Taback B, Leitch AM, Flippo-Morton T, Hunt KK (2014) Tumor biology correlates with rates of breast-conserving surgery and pathologic complete response after neoadjuvant chemotherapy for breast cancer: findings from the ACOSOG Z1071 (Alliance) prospective multicenter clinical trial. Ann Surg 260(4):608-614 (discussion 614-606)

48. Huo CW, Chew GL, Britt KL, Ingman WV, Henderson MA, Hopper JL, Thompson EW (2014) Mammographic density-a review on the current understanding of its association with breast cancer. Breast Cancer Res Treat 144(3):479-502

49. Sherratt MJ, McConnell JC, Streuli CH (2016) Raised mammographic density: causative mechanisms and biological consequences. Breast Cancer Res 18(1):45

50. Bae MS, Moon HG, Han W, Noh DY, Ryu HS, Park IA, Chang JM, Cho N, Moon WK (2016) Early stage triple-negative breast cancer: imaging and clinical-pathologic factors associated with recurrence. Radiology 278(2):356-364

51. Skarping I, Fornvik D, Sartor H, Heide-Jorgensen U, Zackrisson S, Borgquist S (2019) Mammographic density is a potential predictive marker of pathological response after neoadjuvant chemotherapy in breast cancer. BMC Cancer 19(1):1272

52. Elsamany S, Alzahrani A, Abozeed WN, Rasmy A, Farooq MU, Elbiomy MA, Rawah E, Alsaleh K, Abdel-Aziz NM (2015) Mammographic breast density: predictive value for pathological response to neoadjuvant chemotherapy in breast cancer patients. Breast 24(5):576-581

53. Bertrand KA, Tamimi RM, Scott CG, Jensen MR, Pankratz V, Visscher D, Norman A, Couch F, Shepherd J, Fan B et al (2013) Mammographic density and risk of breast cancer by age and tumor characteristics. Breast Cancer Res 15(6):R104

54. van la Parra RF, Peer PG, Ernst MF, Bosscha K (2011) Meta-analysis of predictive factors for non-sentinel lymph node metastases in breast cancer patients with a positive SLN. Eur J Surg Oncol 37(4):290-299

55. Majid S, Ryden L, Manjer J (2019) Determinants for non-sentinel node metastases in primary invasive breast cancer: a populationbased cohort study of 602 consecutive patients with sentinel node metastases. BMC Cancer 19(1):626

56. Zhang L, Liu C, Wang W, Xu X, Chen B (2012) Is optimal timing of sentinel lymph node biopsy before neoadjuvant chemotherapy in patients with breast cancer? A literature review. Surg Oncol 21(4):252-256

57. Zetterlund L, Celebioglu F, Axelsson R, de Boniface J, Frisell J (2017) Swedish prospective multicenter trial on the accuracy and 
clinical relevance of sentinel lymph node biopsy before neoadjuvant systemic therapy in breast cancer. Breast Cancer Res Treat 163(1):93-101

58. Ashikaga T, Krag DN, Land SR, Julian TB, Anderson SJ, Brown AM, Skelly JM, Harlow SP, Weaver DL, Mamounas EP et al (2010) Morbidity results from the NSABP B-32 trial comparing sentinel lymph node dissection versus axillary dissection. J Surg Oncol 102(2):111-118

59. Krag DN, Anderson SJ, Julian TB, Brown AM, Harlow SP, Costantino JP, Ashikaga T, Weaver DL, Mamounas EP, Jalovec LM et al (2010) Sentinel-lymph-node resection compared with conventional axillary-lymph-node dissection in clinically nodenegative patients with breast cancer: overall survival findings from the NSABP B-32 randomised phase 3 trial. Lancet Oncol 11(10):927-933
60. Eisenhauer EA, Therasse P, Bogaerts J, Schwartz LH, Sargent D, Ford R, Dancey J, Arbuck S, Gwyther S, Mooney M et al (2009) New response evaluation criteria in solid tumours: revised RECIST guideline (version 1.1). Eur J Cancer 45(2):228-247

61. Pyo JS, Jung J, Lee SG, Kim NY, Kang DW (2020) Diagnostic accuracy of fine-needle aspiration cytology and core-needle biopsy in the assessment of the axillary lymph nodes in breast cancer-a meta-analysis. Diagnostics (Basel) 10(9):717

Publisher's Note Springer Nature remains neutral with regard to jurisdictional claims in published maps and institutional affiliations. 